\title{
VEINTICINCO AÑOS DE FORMACIÓN DEL GOBIERNO
}

MIGUEL REVENGA

Universidad de Cádiz 



\title{
VEINTICINCO AÑOS DE FORMACIÓN DEL GOBIERNO
}

\author{
POR \\ MIGUEL REVENGA \\ Universidad de Cádiz
}

1. Al redactar cualquier norma jurídica, se supone que es voluntad de sus autores establecer disposiciones claras, capaces de regular satisfactoriamente aquella parcela de la realidad social hacia el que van dirigidas. Aunque es bien conocida la reticencia con la que los británicos contemplan la posibilidad de asomarse al modo mediante el cual son fabricadas las salchichas y las leyes, bien podemos dar por descontado que la oscuridad o la ambigüedad de los mandatos normativos no están nunca entre los objetivos conscientes de los legisladores. Ni siquiera en un ámbito como el de la norma constitucional, "marco de coincidencias lo suficientemente amplio (...)", como para que las imprecisiones, aún buscadas de propósito, puedan ser tenidas por virtuosa disposición hacia el acuerdo.

Cuando se trata de medir, al cabo de los años, el grado en que la labor del legislador ha tenido éxito, puede dudarse de cuáles sean los indicadores apropiados para tener en cuenta; pero es seguro que un par de factores han de ser considerados necesariamente. El primero es el más simple de todos, y atañe a la pura permanencia en el tiempo de la norma, tal y como fue urdida por quienes la redactaron. El segundo, ya de por sí más complejo, se refiere (por decirlo en la jerga de sociológica al uso) a la "tasa de conflictividad" experimentada por la norma en cuestión. Para intentar aclarar lo que entiendo por semejante tasa, proclamo de inmediato mi renuncia a conferir al fac- 
tor cualquier apariencia de objetividad contable. Mis pocas luces de hombre de letras llegan a discernir que aquellas normas concebidas para ser aplicadas en unos pocos supuestos - las de más limitado ámbito normativo - tendrían en el punto de partida una ventaja insalvable con respecto a aquellas de alcance indeterminado y aplicación cotidiana. Diré entonces que la tasa de conflictividad es mi particular criterio subjetivo para medir no ya el número de sentencias o resoluciones judiciales recaídas sobre una determinada norma, sino la capacidad de ésta para continuar cumpliendo por sí misma los objetivos, por naturaleza confesables, que el legislador en su día le asignara. Por sí misma significa "según su letra", lo que equivale a repudiar, en este exigente contexto, las alabanzas de ley que pretextan menosprecios de intenciones: aquello de que «la ley es más inteligente que el legislador" suele ser mera fórmula de cortesía para no decir sin ambages que inteligente, lo que se dice inteligente, es el intérprete.

Una baja tasa de conflictividad de una norma puede denotar falta de aplicación efectiva, o irrelevancia. Pero puede ser también indicio de buena factura, $y$ de capacidad de supervivencia al margen de ( $y$ en muchos casos, frente a) añadidos jurisprudenciales o disposiciones de desarrollo. Una norma no es conflictiva, en suma, si una lectura avezada de la misma no necesita adentrarse, para ser entendida cabalmente, por los vericuetos de las re- (o de-) construcciones interpretativas realizadas por los "señores del derecho" de cada momento.

2. El artículo 99 es una de las disposiciones de la Constitución que suele aparecer en los repertorios anotados huérfano de referencias legislativas o jurisprudenciales. Y ello no por descuido de los autores de tales repertorios, sino porque la letra del artículo se nos aparece en términos tan diáfanos, que es difícil concebir conflictos, a propósito de su aplicación, que no sean de aquel tipo que solemos calificar desdeñosamente como "hipótesis de laboratorio". No suele haber referencias, sencillamente, porque nada hay que reseñar: ni desarrollos legislativos, porque la norma es autosuficiente, ni jurisprudencia interpretativa, porque la tasa de conflicto de la norma equivale a cero. Y sin embargo tengo para mi que el "laboratorio», este sí cierto y comprobable, de las aplicaciones del artículo, ha dado lugar a una serie de prácticas que, si no se resaltan lo suficiente, es porque la experiencia de vida constitucional incorporada al mismo es una experiencia de buen funcionamiento y óptima valía con respecto al fin para el que fue concebido, y no una de vacíos, oscuridades y conflictos.

3. En el artículo 99 de la Constitución está, como es sabido, el núcleo del procedimiento para la sustitución del Gobierno cesante por 
un nuevo Gobierno en plenitud de atribuciones. El grueso de su contenido atañe al procedimiento parlamentario para la instauración de un vínculo de confianza entre el Congreso de los Diputados y el Presidente del Gobierno. Es sobre todo una norma de Derecho parlamentario en sentido material, pero cuyo contenido excede el que es propio de éste, ya que también desarrolla la forma de ejercicio de la función regia prevista en el artículo 62. d) de la Constitución. Fuera de él queda, sin embargo, uno de los actos de impulso capitales para culminar el procedimiento de formación del nuevo Gobierno: el que consiste en la propuesta, a cargo de su Presidente, y ulterior nombramiento por el Rey, de quienes pasarán a ser miembros de dicho órgano colegiado.

El primer acierto es haber dado con la fórmula como para que cualquiera de los supuestos de cese del Gobierno contemplados en el artículo $101.1 \mathrm{CE}$, actúe a modo de espoleta para poner en marcha un procedimiento reglado, enmarcado en límites temporales bastante precisos, y con un "horizonte" constitucional que lleva necesariamente a la alternativa de un nuevo Gobierno en condiciones de atender sus funciones, o la convocatoria de elecciones generales. Una tradición política dada a resolver en falso las interinidades del poder, por la vía del puro falseamiento del proceso democrático, o por ahogo pasional - a menudo solemnizado en altisonantes sesiones parlamentarias- de las posibilidades de concordia, es lógico que apreciara el atractivo de un modelo de racionalización plural en sus objetivos, y satisfactorio a priori para casi todos.

4. Como no albergo duda de que, entre los miembros de la Ponencia, eran mayoría los que conocían, y habían leído a MirkineGuetzevitch, estoy convencido de que la obra del gran clásico de la racionalización del poder durante el período de entreguerras, fue una de las fuentes implícitas de la fórmula. Aunque lo que nos consta fehacientemente es que el reflejo que el constituyente quiso ver al mirar en el espejo del Derecho comparado, fue la muy estable democracia cancilleral de la todavía llamada República Federal de Alemania. Ni que decir tiene que estabilidad gubernamental, estabilidad política y consolidación democrática eran en aquella sazón una y la misma cosa; algo justificado, por lo demás, pues 1978, que fue el año del asesinato de Aldo Moro, no fue precisamente un tiempo favorable para las sutilezas académicas del soppravivere senza Governo. Todo el andamiaje de nuestro artículo 99 es reconocible en el artículo 63 de la Constitución alemana, aunque quizá no se ha resaltado lo suficiente, ni yo mismo supe hacerlo en su momento, que este último artículo, al repudiar cualquier posibilidad de debate, configura un papel de la Dieta 
Federal en la elección de Canciller que está mucho más próximo al preconizado por Bagehot para la Cámara de los Comunes, de lo que lo está el Congreso de los Diputados durante el desarrollo de la sesión de investidura.

5. Planeara o no el fantasma del autor ruso-francés sobre la cabeza de los reunidos en Gredos, lo cierto es que la suprema expresión del proceso racionalizador, cuyo ejemplo quiso ver Mirkine en aquellos textos que cargaron sobre el Parlamento la plena responsabilidad de elegir Gobierno, sin impulso ni mediación alguna supra partes, no era algo que estuviera al alcance de nuestros «Padres fundadores». Con el artículo 99 se logró, sin embargo, cuadrar el círculo de las dos exigencias contradictorias agrupadas bajo la definición de la forma política del Estado del artículo 1.2: las derivadas de una sedicente tradición monárquica y las procedentes de una triunfante razón democrática. Conforme a aquélla, es atributo irrenunciable del Rey el nombramiento de los miembros de la "parte subalterna» del Poder Ejecutivo. Conforme a la razón democrática, ninguna legitimidad distinta de la que deriva de la voluntad del cuerpo electoral - por mediación de los partidos, habría que añadir, pero ése es otro tema- es válida a la hora de formar Gobierno. Lo que el artículo 99 tiene de diáfano se inspira en tal razón, trocada aquí en una lógica de cálculos numéricos e imperativos temporales: un (prescindible) desideratum de mayoría absoluta vinculado a una solución alternativa, y la posibilidad de realizar tantos intentos de investidura como sea posible en el plazo de dos meses contados desde el fracaso de la primera votación. Eso además de un requisito sustancial para el desarrollo de la sesión, a saber: la necesidad de que el candidato defienda ante el Pleno el programa del Gobierno, de cuya gestación se trata; un requisito que otorga, como decimos, al artículo un sesgo peculiar en el panorama comparado.

6. En contraste con esas disposiciones de clarísimo tenor, se nos aparece en el artículo una zona de penumbra, que es característica de muchas de las disposiciones relacionadas con el papel de la Corona, y que vuelve a evocarnos otra famosa aportación de Bagehot a la teoría constitucional: la que diferencia entre efficient y dignified parts of the Constitution. Los fragmentos dignified alcanzan quizá su mayor grado de intensidad en el artículo que abre el Título II, y señaladamente en su Primer párrafo, del que todo lo demás, incluido lo que los artículos 99 y 100 disponen con respecto al Rey, procede. En esa zona de penumbra, la mayor parte de lo que acertemos a decir será siempre aproximativo, y sujeto a la cláusula rebus sic stantibus. Ahora bien, una zona de claroscuros es lo que su nombre indica, una mez- 
cla de luces y sombras, pero no un ámbito ciego de imposible acceso. $Y$ por otra parte un stantibus que dura ya veinticinco años lo menos que permite es el intento de elevar la anécdota empírica a categoría teórica.

7. Podemos tener por cierto que el aserto del artículo 99.1 «(...) el Rey, previa consulta con los representantes designados por los Grupos políticos con representación parlamentaria, y a través del Presidente del Congreso, propondrá un candidato a la Presidencia del Gobierno", significaba, en el momento de la escritura constitucional, al menos tres cosas distintas, según las inclinaciones de cada cual con respecto a la institución monárquica. Para unos, genuina atribución propia del Rey, de ejercicio informado de conformidad con los resultados electorales, y con un refrendo certificante por parte del Presidente del Congreso. Para otros, meras formalidades de cortesía constitucional, consecuentes con la posición jurídica del Rey como jefe del Estado, pero carentes por completo de cualquier peso sustantivo. $Y$ para un tercer grupo, en fin, la consecuencia necesaria de la posición del Rey, no ya como jefe jurídico del Estado, sino como árbitro o potencial moderador del funcionamiento regular de las instituciones, en una survivance constantiana que habria encontrado, precisamente aqui, su resquicio de acomodo.

8. Si tuviera que dar mi impresión sobre cuál de esos tres modelos predomina en la doctrina española, no dudaria en señalar el apuntado en tercer lugar. De todas las atribuciones del Rey, la propuesta de candidato a Presidente del Gobierno suele ser considerada como aquella de mayor influjo potencial sobre el proceso político. Quienes así opinan invocan las cláusulas definitorias de la posición del Rey en el sistema, y completan a veces su tesis defendiendo que el refrendo de los actos regios no es una categoría constitucional unívoca, sino parcelable en distintos tipos, según cuál sea el peso sustantivo de refrendador y refrendado en el proceso que lleva a la decisión del acto en cuestión. Todo ello con el añadido de las usuales advertencias acerca del carácter excepcional de tal atributo regio, destinado a mantenerse dormido, a menos que determinadas circunstancias políticas, comprometedoras del buen destino del proceso de formación del Gobierno, lo despierten y aconsejen hacer uso de tal poder de reserva.

En mi tesis doctoral, me separé resueltamente de esta opinión mayoritaria, e intenté demostrar que la posición del Rey en nuestro sistema constitucional era radicalmente incompatible con cualquier intervención de la Corona en la política activa. Y política activa sería necesariamente cualquier iniciativa, reconducible a la persona del Rey, que 
ensayara fórmulas para intentar desbloquear un procedimiento cuyo hito final, para el caso de dificultades insalvables, está ya contemplado en el propio artículo 99.

9. La glosa del artículo 99 que ensayé en mi memoria doctoral tuvo su principal fundamento en unas pocas prácticas aplicativas, 4 para ser exactos, en los apenas 10 años de vida constitucional que pude tener en cuenta. Ahora, con el horizonte temporal ensanchado hasta los 25 años, y con un número de procesos de investidura que dobla exactamente aquellos 4 , sigo suscribiendo, si cabe con más énfasis, lo que afirmé entonces. Una práctica constante, que quizá cabe ya calificar de genuina costumbre constitucional, no aporta razón alguna para sostener que la relación entre el Rey y el Presidente del Congreso, a efectos de carga de la decisión y refrendo, en lo que se refiere al impulso del procedimiento mediante la propuesta de candidato, tenga alguna característica que haga de ella algo único en el conjunto de las facultades que el Rey tiene atribuidas en nuestro sistema constitucional. O por decirlo de forma menos barroca: la organización del procedimiento de investidura parece concebida para poner al Rey a resguardo de que su facultad de propuesta, "a través del Presidente del Congreso", suponga para él un riesgo de que aquélla sea interpretada como un gesto de apoyo, o una decantación, en favor de una concreta fórmula de gobierno.

10. Cuando escribo estas líneas, en junio de 2003 , la defección de la disciplina de grupo por parte de dos diputados de la Asamblea de Madrid, está abriendo unas incógnitas de cara a la formación de Gobierno, en esa Comunidad Autónoma, que probablemente lleven a una disolución anticipada de la Cámara como consecuencia de la aplicación de la disposición autonómica "gemela" del artículo 99. A la vista de ello, lo que cabe preguntarse es si, sobre una situación política tan complicada, pudiera tener algún efecto el disponer de un fondo de reserva de poder neutral, apto para propiciar acuerdos. Seguramente la pregunta es retórica, pero no creo que sea ocioso recordar que los 17 subsistemas parlamentarios de gobierno, sobre los que pivota nuestro modelo de descentralización política funcionan, con mayor o menor estabilidad según la correlación de fuerzas políticas de cada momento, sin instancia disponible para "animar" o desaconsejar soluciones a las crisis que puedan presentarse desde el momento mismo en que se trata de formar Gobierno. Y que no se nos objete que el fondo de reserva ante esa concreta tesitura es prerrogativa sustancial del modelo de constitucionalismo monárquico; porque entonces lo que cabe decir es que tampoco lo contempla a tales efectos (rectius, lo repudia) una monarquía de tanto peso sobre el propio modelo como la sueca. 
11. En el procedimiento para la formación de Gobierno, el funcionamiento regular de las instituciones significa sopesar dos ideas hasta cierto punto contradictorias. De un lado, la urgencia para poner fin a una situación de interinidad política que nunca es deseable; de otro, la necesidad de coordinar los "calendarios" del Congreso (y los de la Casa del Rey), sabiendo que, desde tal punto de vista, toda el margen de elasticidad que permite el artículo 99 desaparece desde el mismo momento en que queda establecida la fecha para la sesión de investidura. A la vista de lo sucedido hasta ahora, puede comprobarse que el tracto típico de los acontecimientos (constitución del Congreso, elección de Presidente, consultas regias, propuesta y sesión de investidura) exige un número de dias que ha oscilado entre un mínimo de 26, en 1981 -aunque aquí el supuesto desencadenante fue la dimisión de Adolfo Suárez- y un máximo de 60, en 1996. El Cuadro 1 permite ver el tiempo transcurrido desde las elecciones (o desde la dimisión del Presidente, en 1981) hasta el otorgamiento de la confianza en primera o segunda votación, en las 8 investiduras celebradas entre 1978 y 2003.

CUADRO 1

NÚMERO DE DÍAS INVERTIDOS EN LA REALIZACIÓN DE LOSTRÁMITES PREVISTOS EN EL ARTÍCULO 99 PARA LA INVESTIDURA DEL CANDIDATO A PRESIDENTE DEL GOBIERNO, 1978-2003

\begin{tabular}{lll}
\hline Gobierno & $\begin{array}{l}\text { Fecha/supuesto } \\
\text { desencadenante }\end{array}$ & $\begin{array}{l}\text { Fecha investidura } \\
\text { (Número de días) }\end{array}$ \\
\hline Suárez II & Elecciones 01/03/1979 & $30 / 03 / 1979(27)$ \\
\hline Calvo Sotelo & Dimisión 29/01/1981 & $25 / 02 / 1981(26)$ \\
\hline González I & Elecciones 28/10/1982 & $01 / 12 / 1982(33)$ \\
\hline González II & Elecciones 22/06/1986 & $23 / 07 / 1986(29)$ \\
\hline González III & Elecciones 29/10/1989 & $05 / 12 / 1989(36)$ \\
\hline González IV & Elecciones 06/06/1993 & $09 / 07 / 1993(31)$ \\
\hline Aznar I & Elecciones 03/03/1996 & $04 / 05 / 1996(60)$ \\
\hline Aznar II & Elecciones 12/03/2000 & $26 / 04 / 2000(43)$ \\
\hline
\end{tabular}

Fuente: elaboración propia. 
En materia de calendario, la sensibilidad política del Presidente del Congreso resulta esencial. Auxiliado por los órganos rectores de la Cámara, a él le corresponde fijar el orden del día, pero sólo sobre él (o sobre ella) recae la responsabilidad de "interpretar" el curso de las hipotéticas negociaciones entre las fuerzas políticas para decidir el cuándo de la investidura. Y no sólo el cuándo de la investidura; también el Presidente del Congreso, cuya función en el proceso nunca se ha interpretado como meramente ancilar de la del Rey, ha de desplegar buenas dosis de tino político para aconsejar a éste quién debe ser llamado a las consultas, así como, llegado el caso, para propiciar un nuevo turno de ellas, o bien acelerar los trámites que lleven a la convocatoria de nuevas elecciones.

$Y$ en relación con las consultas, todavía un comentario. Excepto en la investidura de 1981, en la que la selección de los consultados se realizó sobre la base de los grupos parlamentarios del Congreso, en todas las demás lo que parece prevalecer es el deseo de que el Rey tenga contacto institucional con todas y cada una de las fuerzas políticas que han obtenido escaño en dicha Cámara (Cuadro 2). La cuestión no es baladí, pues si las cosas continúan siendo como hasta ahora, es difícil sostener que el objeto inmediato de las consultas sea realizar la propuesta más viable. Entre otras razones que tienen que ver con el propio modo de realizarlas, porque la postura de cada fuerza se conoce de antemano, o bien, como ocurrió en 1996 durante el proceso de negociación entre el PP y CiU, porque resulta todavía incierta cuando el representante es llamado a expresar a su parecer.

No deja de ser curioso que en 1993 apareciera en la lista de consultados $-y$ acudiera a La Zarzuela - el portavoz de Herri Batasuna, Jon Idígoras, pese a la actitud de boicot institucional mantenida sistemáticamente por dicho grupo político. También nos parece digno de mención (aunque no sabríamos decir a qué obedece el cambio) el hecho de que, a partir de dicho año, el orden cronológico de realización de las consultas se ha invertido en relación con el que había predominado hasta entonces: el de la fuerza parlamentaria, ordenada de mayor a menor, del partido o grupo político de cada uno de los consultados. 
CUADRO 2

CONSULTAS PREVIAS A LA PROPUESTA DE CANDIDATO A PRESIDENTE DEL GOBIERNO (1978-2003) $(*)$

\begin{tabular}{|c|c|c|c|}
\hline \multicolumn{2}{|l|}{1979} & \multicolumn{2}{|l|}{1981} \\
\hline Líderes consultados & $\begin{array}{c}\text { Fuerza } \\
\text { parlam. }\end{array}$ & Lideres consultados & $\begin{array}{l}\text { Fuerza } \\
\text { parlam }\end{array}$ \\
\hline Adolfo Suárez (UCD) & 168 & Adolfo Suárez (UCD) & 165 \\
\hline Felipe González (PSOE) & 121 & Felipe González (PSOE) & 119 \\
\hline Santiago Carrillo (PCE) & 23 & Santiago Carrillo (PCE) & 3 \\
\hline Manuel Fraga (CD) & 9 & Manuel Fraga (CD) & 9 \\
\hline Jordi Pujol (CiU) & 8 & Blas Piñar (UN) & 1 \\
\hline Carlos Garaicoechea (PNV) & 7 & Alejandro Rojas (PSA) & 5 \\
\hline Blas Piñar (UN) & 1 & Juan M. Bandrés (EE) & 1 \\
\hline H. G. de las Roces (PAR) & 1 & Marcos Vizcaya (PNV) & 7 \\
\hline Juan M. Bandrés (EE) & 1 & Miguel Roca (CiU) & 9 \\
\hline Javier Aizpún (UPN) & 1 & & \\
\hline Fernando Sagaseta (UPC) & 1 & & \\
\hline Heribert Barrera (ERC) & 1 & & \\
\hline Joan Raventós (PSC-PSOE) & 1 & & \\
\hline \multicolumn{2}{|l|}{1982} & \multicolumn{2}{|l|}{1986} \\
\hline Felipe González (PSOE) & 202 & Miguel Izquierdo (UV) & 1 \\
\hline Manuel Fraga (CP) & 107 & H. G. de las Roces (PAR) & 1 \\
\hline Landelino Lavilla (UCD) & 11 & Senén Bernárdez (CG) & 1 \\
\hline Miguel Roca (CiU) & 8 & Manuel Hermoso (AIC) & 1 \\
\hline Javier Arzallus (PNV) & 8 & Juan M. Bandrés (EE) & 2 \\
\hline Santiago Carrillo (PCE) & 4 & Iñaqui Anasagasti (PNV) & 6 \\
\hline Adolfo Suárez (CDS) & 2 & Gerardo Iglesias (IU) & 7 \\
\hline Francesc Vicens (ERC) & 1 & Miguel Roca (CiU) & 18 \\
\hline \multirow[t]{3}{*}{ Juan M. Bandrés (EE) } & 1 & Adolfo Suárez (CDS) & 19 \\
\hline & & Manuel Fraga (CP) & 105 \\
\hline & & Felipe González (PSOE) & 184 \\
\hline
\end{tabular}


CUADRO 2 (continuación)

CONSULTAS PREVIAS A LA PROPUESTA DE CANDIDATO A PRESIDENTE DEL GOBIERNO (1978-2003) (*)

\begin{tabular}{lcllc}
\hline \multicolumn{1}{c}{1989} & & \multicolumn{2}{c}{1993} & \\
\hline Lideres consultados & $\begin{array}{c}\text { Fuerza } \\
\text { parlam. }\end{array}$ & Lideres consultados & $\begin{array}{c}\text { Fuerza } \\
\text { parlam. }\end{array}$ \\
\hline Felipe González (PSOE) & 166 & V. González Lizondo (UV) & 1 \\
\hline José María Aznar (PP) & 105 & Xavier Albistur (EA) & 1 \\
\hline Julio Anguita (IU) & 17 & José María Mur (PAR) & 1 \\
\hline Adolfo Suárez (CDS) & 13 & Pilar Rahola (ERC) & 1 \\
\hline Miguel Roca (CiU) & 18 & Jon Idígoras (HB) & 2 \\
\hline Iñaki Anasagasti (PNV) & 5 & Lorenzo Olarte (CC) & 4 \\
\hline V. González Lizondo (UV) & 2 & Iñaki Anasagasti (PNV) & 5 \\
\hline A. Rojas Marcos (PA) & 1 & Miguel Roca (CiU) & 17 \\
\hline Joseba Azcárraga (EA) & 2 & Julio Anguita (IU) & 17 \\
\hline Jon Larrinaga (EE) & 1 & José María Aznar (PP) & 141 \\
\hline José María Mur (PAR) & 1 & Felipe González (PSOE) & 159 \\
\hline Luis Mardones (AIC) & 1 & & & \multicolumn{1}{c}{2000} \\
\hline \multicolumn{1}{c}{1996} & & & & \\
\hline José María Chiquillo (UV) & 1 & José A. Labordeta (ChA) & 1 \\
\hline Begoña Lasagabaster (EA) & 1 & Begoña Lasagabaster (EA) & 1 \\
\hline Pilar Rahola (ERC) & 1 & Joan Saura (IC-V) & 1 \\
\hline Francisco Rodríguez (BNG) & 2 & Joan Puigcercós (ERC) & 1 \\
\hline José Carlos Mauricio (CC) & 4 & José Núñez (PA) & 1 \\
\hline Iñaki Anasagasti (PNV) & 5 & Francisco Rodríguez (BNG) & 3 \\
\hline Joaquim Molins (CiU) & 16 & José Carlos Mauricio (CC) & 4 \\
\hline Julio Anguita (IU) & 21 & Iñaki Anasagasti (PNV) & 7 \\
\hline Felipe González (PSOE) & 141 & Francisco Frutos (IU) & 8 \\
\hline José María Aznar (PP) & 156 & Xavier Trias (CiU) & 15 \\
\hline & & Manuel Chaves (PSOE) & 125 \\
\hline & & José María Aznar (PP) & 183 \\
\hline
\end{tabular}

(*) Por orden de realización de la consultas.

Fuente: elaboración propia. 
12. Con la experiencia que hemos acumulado hasta ahora, queda confirmado un rasgo que la doctrina ha venido apuntando desde la aprobación de la Constitución: el artículo 99 incentiva la formación de Gobiernos minoritarios, y además los protege mediante un diseño de la moción de censura, que no es exagerado calificar de "disuasorio". En tales condiciones, la exigencia de mayoría absoluta en primera votación bien puede compararse, a efectos prácticos, a aquella famosa exhortación constitucional a ser justos y benéficos. $\mathrm{Si}$ los efectos combinados de unas determinadas pautas de comportamiento de los votantes, y unos componentes de la ley electoral (principalmente el tipo de circunscripción y el modo de escrutinio) permiten fundamentar ciertos pronósticos nada favorables a la repetición de mayorías absolutas de un solo partido, la opción del artículo 99 se nos sigue apareciendo, al cabo de los años, como la más racional. Sobre todo, si constatamos que los 8 Gobiernos formados hasta el momento han disfrutado de una estabilidad relativamente alta, pese a que sólo la mitad de ellos (los formados por Felipe González en 1982, 1986 y 1989, y por José María Aznar en 2000) han sido, estrictamente hablando, de carácter mayoritario. Y ello manejando un criterio de clasificación flexible, puesto que en 1989, que fue el año en el que hubo que repetir las elecciones en las circunscripciones de Melilla, Murcia y Pontevedra, el cálculo de la mayoría absoluta se realizó, según Resolución al efecto del Presidente del Congreso, sobre un total de 332 escaños; es decir, descontando de los 350 el total de 18 escaños que estaba en liza en tales circunscripciones. El resto de los Gobiernos han sido - conforme a las categorías habituales entre los politólogos - bien minoritarios sustantivos (casos de los Gobiernos Suárez II, y Cavo Sotelo), bien minoritarios formales (casos de los Gobiernos González IV y Aznar I), esto es, "reforzados» mediante pactos de legislatura con fuerzas nacionalistas, que garantizaron la estabilidad. La duración y el tipo de cada uno de tales Gobiernos aparecen recogidos en el Cuadro 3. 
CUADRO 3

ESTABILIDADYTIPOS DE GOBIERNO FORMADOS

1978-2003

\begin{tabular}{lccc}
\hline Gobierno & $\begin{array}{c}\text { Fecha de } \\
\text { investidura }\end{array}$ & $\begin{array}{c}\text { Duración } \\
\text { (días) (meses) }\end{array}$ & $\begin{array}{c}\text { Tipo de } \\
\text { Gobierno }\end{array}$ \\
\hline Suárez II & $30-03-79$ & 66522,1 & UCD (min.) \\
\hline Calvo-Sotelo & $25-02-81$ & 61420,4 & UCD (min.) \\
\hline González I & $1-12-82$ & $1.29943,3$ & PSOE (may.) \\
\hline González II & $23-07-86$ & $1.19039,6$ & PSOE (may.) \\
\hline González III & $5-12-89$ & $1.27942,6$ & PSOE (may.) \\
\hline González IV & $9-7-93$ & 99733,2 & PSOE (min.) \\
\hline Aznar I & $4-5-96$ & $1.32544,2$ & PP (min.) \\
\hline Aznar II & $26-04-00$ & - & PP (may.) \\
\hline
\end{tabular}

Fuente: Adaptado de Josep Maria Reniu Villamala, “ ¿Merece la pena coaligarse? La formación de Gobiernos minoritarios en España, 1977-1996", Revista Española de Ciencia Política, 5 (2001), págs. 111 y ss.

13. El hecho de que en los 25 años transcurridos desde la aprobación del texto constitucional desconozcamos la experiencia de Gobiernos formados por más de un partido - caso único en el seno de lo que suele llamarse los países de nuestro entorno- no permite comprobar nada distinto a un refuerzo extraordinario de la figura del Presidente del Gobierno en nuestro sistema. Con un estatuto constitucional ya de por sí muy realzado con respecto al resto de los miembros del Gobierno, el artículo 100 propicia que en la formación de Gobierno el Presidente pueda comportarse como un monarca del Antiguo Régimen, nombrando y destituyendo consejeros à son plaisir. Bajo la vigencia de la Ley 10/1983, de organización de la Administración Central del Estado, vimos cómo claudicaba la aspiración constitucional a que el principio de legalidad rigiera algo tan esencial como la estructura interna del Gobierno. $Y$ tal deslegalización se ha visto plenamente consumada en el artículo 2 de la Ley 50/1997, del Gobierno, donde la creación, modificación o supresión de los Departamentos, mediante Real Decreto, aparece así definida; sin circunloquios, como atribución propia del Presidente.

El principio de dirección presidencial, que alcanza, según la ley de 1997, al establecimiento del programa político del Gobierno (artículo 2.2 b), se vería sin duda atenuado en las condiciones políticas de 
un Gobierno de coalición. El funcionamiento de los subsistemas políticos autonómicos, donde hasta mayo de 2003, 31 de los 112 Gobiernos formados desde 1980 (es decir, un porcentaje nada desdeñable del 27,67 por ciento) han sido de tal carácter, ofrece al respecto un buen laboratorio de pruebas. Pero con independencia de ello, lo que hasta hoy sabemos es que la investidura del artículo 99 de la Constitución, bascula sobre la persona de un candidato que debate con todos acerca de su programa, y que reserva celosamente para sí la facultad de diferir a un momento posterior al de la intervención del Congreso la decisión relativa a la composición personal (y a la estructura formal) del Gobierno.

14. $Y$ un último apunte; es improbable que suceda, pero si el procedimiento previsto en el artículo 99 desembocara en una disolución anticipada de las Cortes Generales, el hecho de que el Senado se viera afectado por ella, representaría quizá el "golpe de gracia" definitivo para una Cámara lastrada por las inconsecuencias de las definiciones constitucionales y los tabúes en torno a una reforma de la Constitución que las celebraciones del vigésimo quinto aniversario hacen todavía menos factible. 
RESULTADOS DE LAS VOTACIONES DE INVESTIDÚRA, 1978-2003




APÉNDICE

RESULTADOS DE LAS VOTACIONES DE INVESTIDURA, 1978-2003 (continuación)

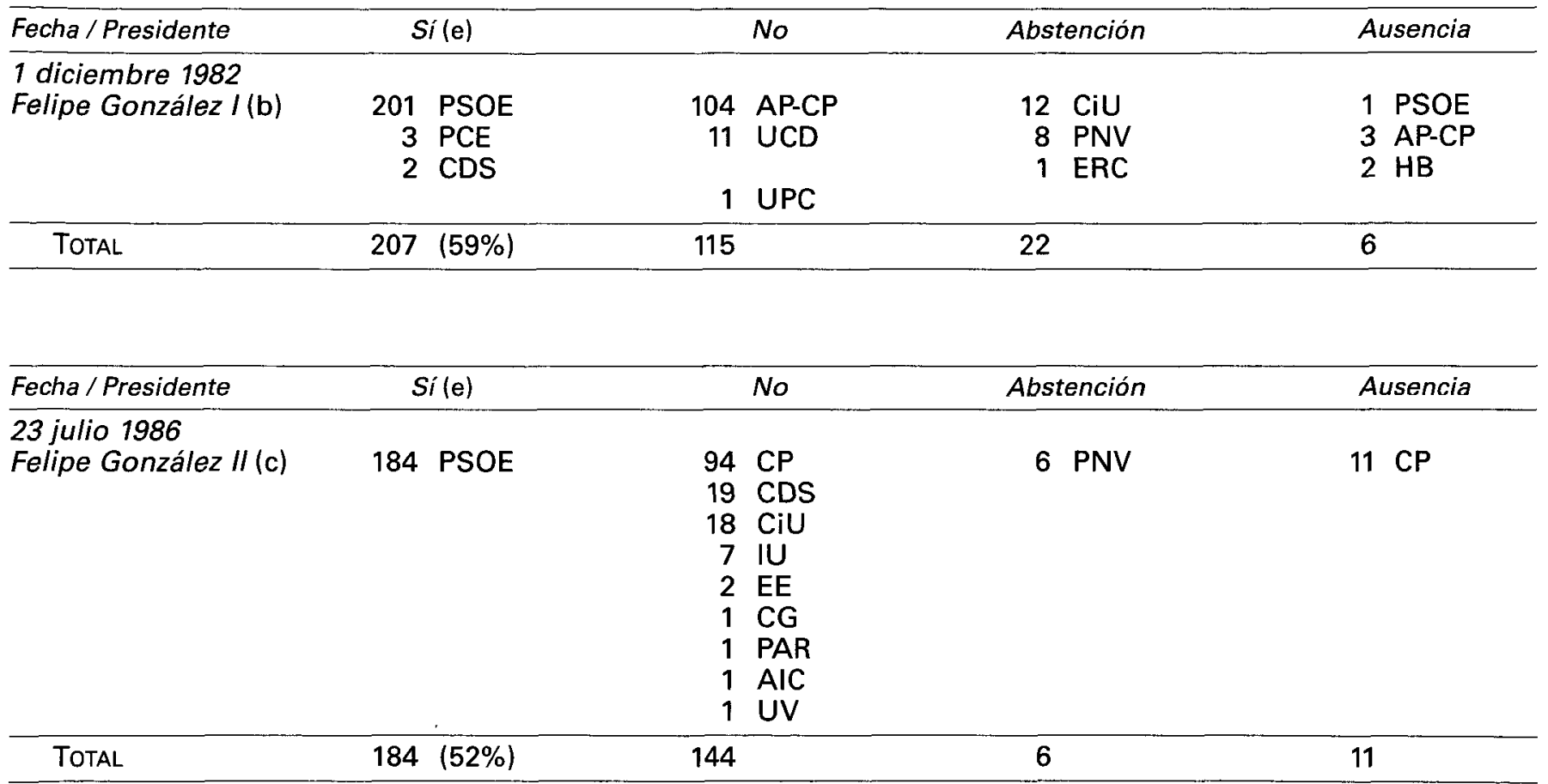




\section{APÉNDICE}

RESULTADOS DE LAS VOTACIONES DE INVESTIDURA, 1978-2003 (continuación)

\begin{tabular}{|c|c|c|c|c|c|}
\hline Fecha / Presidente & Sí(e) & & No & Abstención & Ausencia \\
\hline $\begin{array}{l}5 \text { diciembre } 1989 \\
\text { Felipe González III (d) }\end{array}$ & $\begin{aligned} 166 & \text { PSOE } \\
1 & \text { AIC }\end{aligned}$ & $\begin{array}{r}99 \\
18 \\
17 \\
13 \\
2 \\
2 \\
2 \\
2\end{array}$ & $\begin{array}{l}\text { PP } \\
\text { CiU } \\
\text { IU } \\
\text { CDS } \\
\text { UV } \\
\text { EA } \\
\text { PA } \\
\text { EE }\end{array}$ & $\begin{array}{ll}5 & \text { PNV } \\
1 & \text { PAR }\end{array}$ & $4 \mathrm{HB}$ \\
\hline TOTAL & $167 \quad(51 \%[48 \%])$ & 155 & & 6 & 4 \\
\hline Fecha / Presidente & Si $(\mathrm{e})$ & & No & Abstención & Ausencia \\
\hline $\begin{array}{l}9 \text { julio } 1993 \\
\text { Felipe González IV }\end{array}$ & $\begin{aligned} 159 & \text { PSOE } \\
17 & \text { CiU } \\
5 & \text { PNV }\end{aligned}$ & $\begin{array}{r}141 \\
17 \\
4 \\
1 \\
1 \\
1\end{array}$ & $\begin{array}{l}\text { PP } \\
\text { IU } \\
\text { CC } \\
\text { EA } \\
\text { ERC } \\
\text { UV }\end{array}$ & 1 PAR & $\begin{array}{ll}1 & \mathrm{IU} \\
2 \mathrm{HB}\end{array}$ \\
\hline TOTAL & $181(52 \%)$ & 165 & & 1 & 3 \\
\hline
\end{tabular}




\begin{tabular}{|c|c|c|c|c|c|}
\hline \multicolumn{6}{|c|}{ APÉNDICE } \\
\hline Fecha / Presidente & Si $(\mathrm{e})$ & & No & Abstención & Ausencia \\
\hline $\begin{array}{l}4 \text { mayo } 1996 \\
\text { José María Aznar I }\end{array}$ & $\begin{array}{rl}156 & \mathrm{PP} \\
16 & \mathrm{CiU} \\
5 & \mathrm{PNV} \\
4 & \mathrm{cc}\end{array}$ & $\begin{array}{r}141 \\
21 \\
2 \\
1 \\
1\end{array}$ & $\begin{array}{l}\text { PSOE } \\
\text { IU } \\
\text { BNG } \\
\text { EA } \\
\text { ERC }\end{array}$ & $1 \mathrm{UV}$ & $2 \mathrm{HB}$ \\
\hline TOTAL & $181(52 \%)$ & 166 & & 1 & 2 \\
\hline Fecha / Presidente & $S i(e)$ & & No & Abstención & Ausencia \\
\hline $\begin{array}{l}26 \text { abril } 2000 \\
\text { José María Aznar II }\end{array}$ & $\begin{array}{rl}183 & \mathrm{PP} \\
15 & \mathrm{CiU} \\
4 & \mathrm{CC}\end{array}$ & $\begin{array}{r}125 \\
8 \\
7 \\
3 \\
1 \\
1 \\
1 \\
1 \\
1\end{array}$ & $\begin{array}{l}\text { PSOE } \\
\text { IU } \\
\text { PNV } \\
\text { BNG } \\
\text { EA } \\
\text { ERC } \\
\text { IC-V } \\
\text { PA } \\
\text { ChA }\end{array}$ & & \\
\hline TOTAL & $202 \quad(58 \%)$ & 148 & & 0 & 0 \\
\hline
\end{tabular}




\section{EXPLICACIÓNY NOTAS DEL APÉNDICE}

(a) Tras las elecciones de junio de 1977, Adolfo Suárez no se sometió a votación de investidura al considerarse su gobierno, formado el 5 de julio, como uno de gestión del período constituyente.

(b) La abstención del PSOE corresponde a la no participación en la votación del presidente del Congreso de los Diputados, Gregorio Peces Barba.

(c) El número total de diputados es de 345, puesto que 5 diputados de $\mathrm{HB}$ no tomaron posesión de sus escaños al negarse a acatar la Constitución.

(d) El número máximo de diputados era 332 al haberse producido la anulación provisional de los resultados electorales en las circunscripciones de Murcia, Pontevedra y Melilla y al no tomar posesión los 4 electos de HB. Una decisión de la Presidencia del Congreso, avalada por la Junta de Portavoces, fijó la mayoría absoluta en 167 diputados, es decir, la mitad más uno de los 332 diputados que habían obtenido la condición de tales en el momento del inicio de la sesión de investidura. El 5 de abril de 1990, y ya con el Congreso formado en su totalidad, F. González presentó una moción de confianza para reforzar su voto de investidura; la moción obtuvo el apoyo de 175 diputados del PSOE y de 1 de CC (por tanto, una mayoría del $50,28 \%$, que pasó a ser funcionalmente absoluta al no haber tomado los electos por HB posesión de sus escaños); votaron en contra 130 diputados y 37 se abstuvieron.

(e) Entre paréntesis, porcentaje de escaños sobre el total del Congreso.

Fuente: Adaptado de Juan J. LiNz, José Ramón Montero y Antonia M. RuIz, "Elecciones y política", de próxima publicación en Albert DE CARRERAS y Xavier TAfunell (eds.), Estadísticas Históricas de España, 1800-2000. 